\title{
Calculating environmental moisture for per-field discrimination of rice crops
}

\author{
T. G. VAN NIEL*, T. R. McVICAR
}

CSIRO Land and Water, PO Box 1666, Canberra, ACT 2601, Australia

\section{H. FANG and S. LIANG}

Department of Geography, University of Maryland, College Park, MD 20742, USA

(Received 10 December 2001; in final form 20 June 2002)

\begin{abstract}
The accuracies of rice classifications determined from density slices of broadband moisture indices were compared to results from a standard supervised technique using six reflective Enhanced Thematic Mapper plus $($ ETM +$)$ bands. Index-based methods resulted in higher accuracies early in the growing season when background moisture differences were at a maximum. Analysis of depth of ETM + band 5 resulted in the highest accuracy over the growing season $(97.74 \%)$. This was more accurate than the highest supervised classification accuracy $(95.81 \%)$, demonstrating the usefulness of spectral feature selection of moisture for classifying rice.
\end{abstract}

\section{Introduction}

Discriminating rice from other crops to a high level of accuracy is commonplace using various clustering algorithms, namely supervised classifiers (e.g. Barbosa et al. 1996). In structured environments, the addition of contextual information can increase accuracy over per-pixel classifications (e.g. per-field classification, Aplin et al. 1999). For the specific case of highly regulated agricultural systems where withincrop phenological differences are small, it is also true that a single spectral feature may capture most between-crop variability. Isolating the times when this occurs could ( $i$ ) simplify the classification procedure considerably, and (ii) possibly increase accuracy further by eliminating superfluous information. In this study, we test the ability of spectral features related to 'environmental' moisture content to discriminate rice and non-rice crops. Environmental, as defined here, describes the mixture of plant canopy and background reflectance, and is used to differentiate the current study from leaf-scale studies.

In the following discussion, formulae are adapted to the mean wavelength (MWL, weighted by bandpass function) of Enhanced Thematic Mapper plus $(\mathrm{ETM}+)$ bands. Three spectral features relating to environmental moisture are used, the Normalized Difference Infrared Index $\left(\mathrm{NDII}=\left(\rho_{835 \mathrm{~nm}}-\rho_{1650 \mathrm{~nm}}\right) /\right.$

*Corresponding author; e-mail: Tom.VanNiel@csiro.au

International Journal of Remote Sensing

ISSN 0143-1161 print/ISSN 1366-5901 online (C) 2003 Taylor \& Francis Ltd

http://www.tandf.co.uk/journals

DOI: $10.1080 / 0143116021000009921$ 
$\left(\rho_{835 \mathrm{~nm}}+\rho_{1650 \mathrm{~nm}}\right)$, Hardisky et al. 1983), the Moisture Stress Index (MSI= $\rho_{1650 \mathrm{~nm}} / \rho_{835 \mathrm{~nm}}$, Hunt and Rock 1989$)$, and the depth of ETM + band $5\left(\mathrm{D}_{1650 \mathrm{~nm}}=\right.$ $1-R^{\prime}$, adapted from Kokaly and Clark 1999). Depth of bands within absorption features has been used to estimate various plant-based properties from hyperspectral remote sensing. Continuum-removed reflectance $\left(R^{\prime}\right)$ is determined for every channel within the absorption feature by dividing reflectance of the channel by the value of a reference, or continuum line forming a 'ceiling' above the entire absorption feature (see Kokaly and Clark 1999). In its most simple case, band-depth analysis requires three bands, two defining the ends of the continuum line and one between the end points to be related to the continuum line. This three-point simplification permits the application of band-depth analysis on broadband imagery. For this study, continua end points are established at the MWL of ETM + bands $4(835 \mathrm{~nm})$ and 7 $(2208 \mathrm{~nm}$ ), allowing for the calculation of the continuum-removed reflectance for the MWL of band $5(1650 \mathrm{~nm})$ using the following equation

$$
R^{\prime}=\frac{\rho_{1650 \mathrm{~nm}}}{\left(\rho_{835 \mathrm{~nm}}(1-c)\right)+\left(\rho_{2208 \mathrm{~nm}} c\right)}
$$

where the constant $\mathrm{c}$ represents the relative position of band 5 to, in this case, band 7

$$
c=\frac{1650-835}{2208-835} \approx 0.59359
$$

Because $\rho_{1650 \mathrm{~nm}}$ changes more rapidly with increasing water than $\rho_{835 \mathrm{~nm}}$ and $\rho_{2208 \mathrm{~nm}}$ (Gausman et al. 1978 , Tucker 1980), $R^{\prime}$ and therefore $D_{1650 \mathrm{~nm}}$ are related to environmental moisture. Figure 1 illustrates this relationship, where $D_{1650 \mathrm{~nm}}$ is greater for flooded rice compared to intermittently irrigated soybeans. The distinction between rice and soybeans is maximized prior to canopy closure (figure 1), where background water and soil signals, respectively, have an opposing affect on $D_{1650 \mathrm{~nm}}$. Temporal variations due to different growth rates and management practices (e.g. date of planting) result in different surfaces, affecting the amount of spectral separability between rice and non-rice crops. In this case, discrimination between rice and soybeans is enhanced early in the growing season with respect to environmental moisture.

\section{Study site}

Coleambally Irrigation Area (CIA), New South Wales, is approximately 95000 ha in size, comprising over 500 farms; fields are large (up to 70 ha) and well maintained. Primary summer crops are rice, maize and soybeans. Timing of generalized surface signals is summarized across the bottom of figure 2. Rice is sown in early October and starts senescing in late February. Maize is sown about the same time as rice, yet matures faster, so it is often well into senescence by the end of February. Soybeans are sown later, emerging from the soil in early December with senescence starting in early-mid March. All three crops are harvested from March-April. As opposed to flooded rice, maize and soybeans are only intermittently irrigated.

\section{Methods and discussion of results}

\subsection{Image pre-processing}

Twelve cloud-free ETM + images were acquired between October 2000 and March 2001. Fine spatial resolution digital aerial photographs ( $1.5 \mathrm{~m}$ pixel size), acquired in early January 2001, were used to geo-reference the ETM + images. Atmospheric correction was then applied to all ETM + images. First, surface 


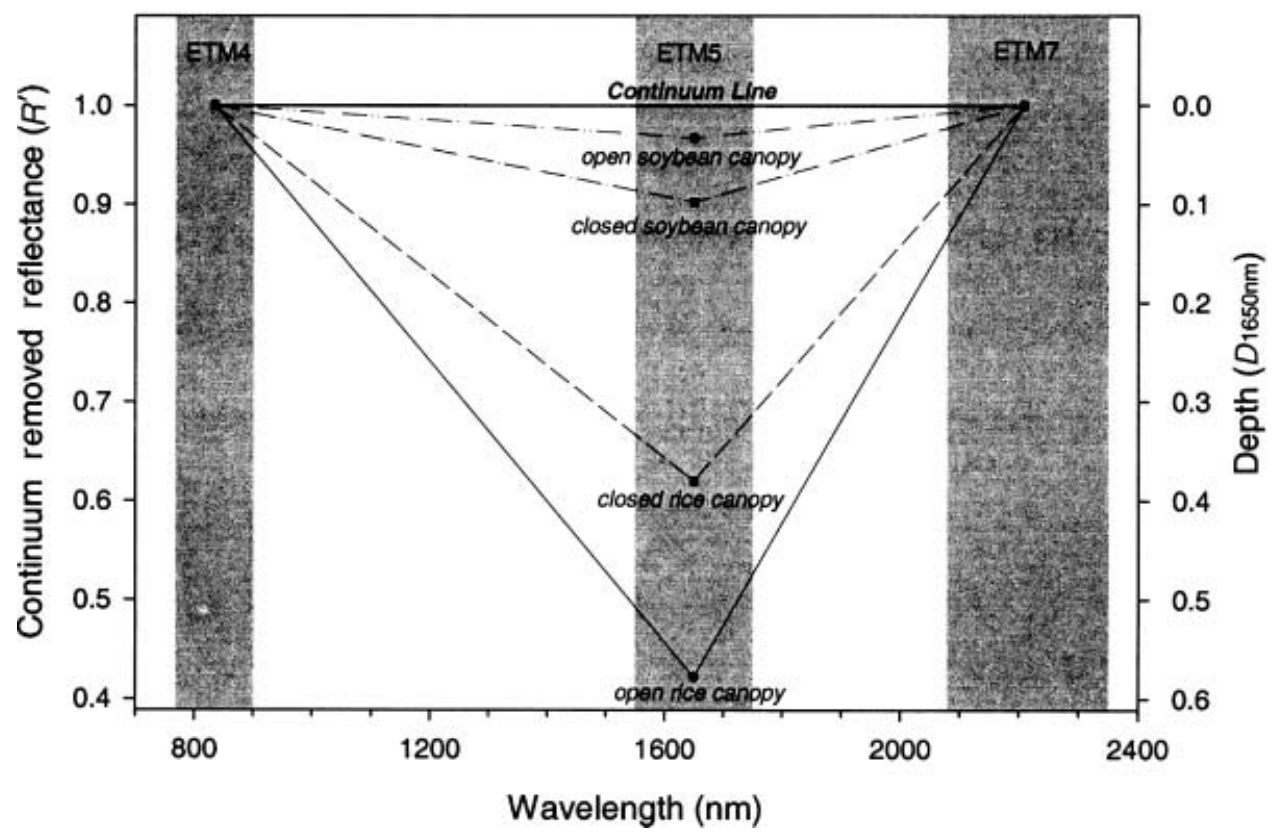

Figure 1. Continuum removed reflectance $\left(R^{\prime}\right)$ and depth of ETM + band $5\left(D_{1650 \mathrm{~nm}}\right)$ for open and closed canopies of rice and soybeans. These ground spectra were collected at the CIA using an Analytical Spectral Devices (ASD) radiometer.

reflectance was calculated for two images when simultaneous ground reflectance was measured ( 3 February and 7 March). Image reflectance was calculated using MODTRAN4 simulations of three atmospheric parameters (path radiance, transmitted flux and spherical albedo) for various atmospheric visibility values. Optimum visibility for clear sky conditions was calculated $(50 \mathrm{~km})$ for these two dates by minimizing the difference between image reflectance and known ground reflectance. Secondly, this optimum visibility value was used to estimate the atmospheric parameters in MODTRAN4 and calculate surface reflectance for the other 10 cloud-free images. Inspection of pseudo invariant features verified the use of this methodology for the 12 images.

Next, $D_{1650 \mathrm{~nm}}$, NDII, MSI and Normalized Difference Vegetation index (NDVI) were calculated for all 12 atmospherically corrected images. Per-field classifications were generated for all 12 dates using two methods: $(i)$ a density slice of each of the previously mentioned indices; and (ii) a maximum likelihood (ML) algorithm using six reflective ETM + bands (excluding the panchromatic band). Vegetated fields were determined from a combination of aerial photographs and a mid-season (3 February) NDVI image. All vegetated fields (1255) were digitized from the photographs and identified as either rice or non-rice. A 310-field subset was used to evaluate the accuracy of all classifications; 155 non-rice fields (verified as NDVI greater than 0.15), and 155 rice fields (arbitrarily, every sixth rice field in the dataset) were selected. This subsampling eliminated unrepresentative results due to the inordinately large proportion of rice fields $(85 \%)$.

Since supervised classification using all reflective bands is standard practice, the ML results serve as a reference to compare the index-based classifications. NDVI is 


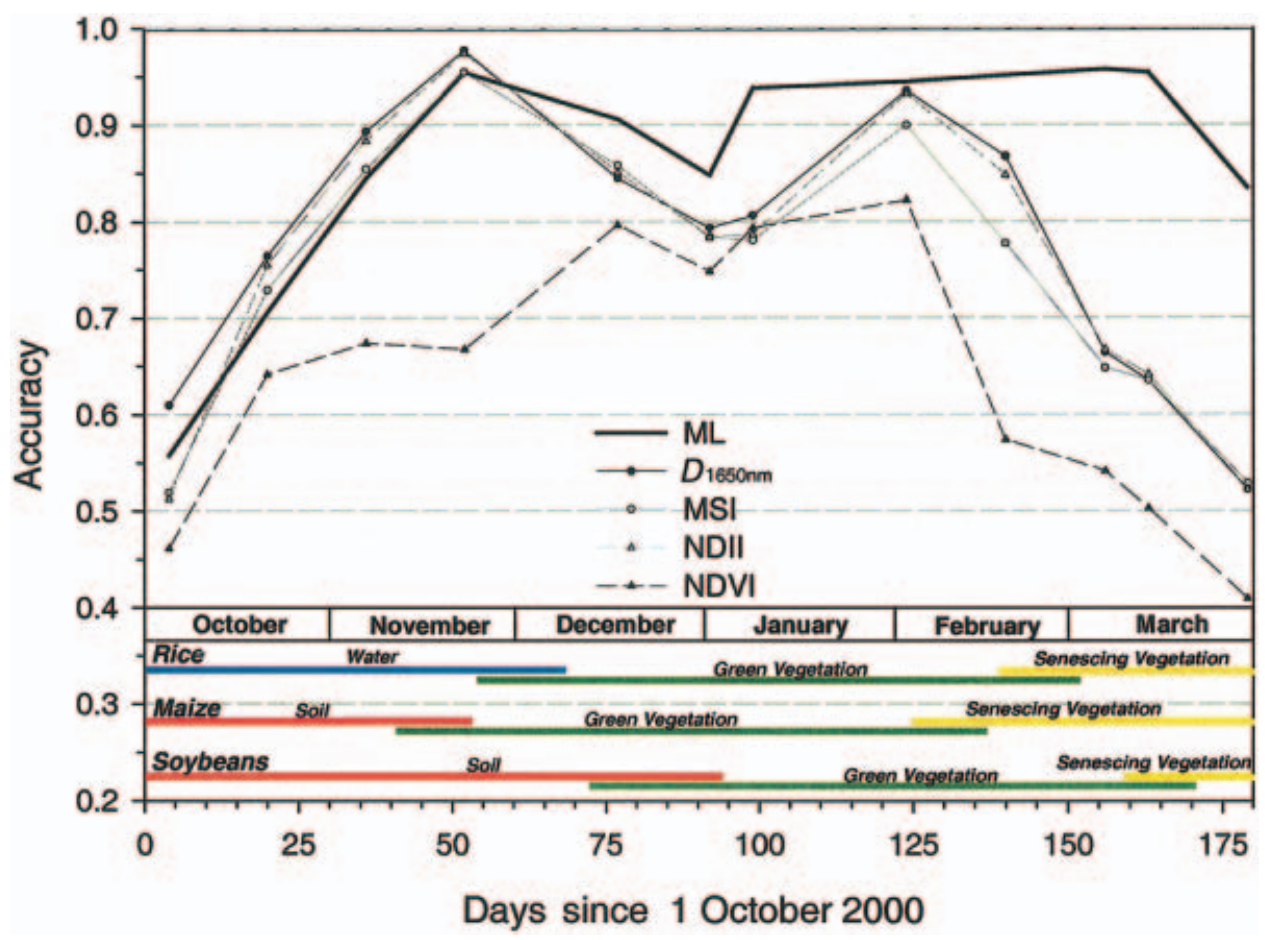

Figure 2. Rice classification accuracies (as proportions) for all dates using both an ML algorithm on six reflective ETM + bands, and a threshold on the indices. A summary of timing of surface signals is represented by coloured lines across the bottom of the figure.

included in all analyses to compare moisture index response to a spectral feature of 'greenness'. All classifications were based on statistics generated from the same training set of five rice (1587 pixels) and five non-rice (2497 pixels) fields. The 10 training set fields were not within the 310-field subset used for accuracy assessment.

\subsection{Discrimination of rice/non-rice}

Mean rice and mean non-rice values were calculated from the 10-field training set for every index/date combination. The average of rice and non-rice means defined the threshold value used to create the density slices for each index. Fields within the 310-field subset with means above the defined threshold were classified as either rice or non-rice (depending on appropriateness for the individual index), while those below were alternately classified. Overall classification accuracy, as defined by Congalton (1991), was calculated, which accounts for errors of omission and commission in an evenly sampled two-by-two error matrix (figure 2).

All three moisture indices demonstrate a 'bimodal' shape, with highest accuracy in late November, followed by a secondary peak in early-mid February. These times highlight phenological variability for the three major crops. In late November, the rice signal was dominated by background water, maize was reaching canopy closure, and soybean fields were bare soil. Conversely, in February, rice was beginning to yellow, maize was almost fully senesced, and soybeans were actively growing. NDVI demonstrated a different response, where highest discrimination occurred mid-season 
when crops were green (figure 2). Maximum accuracy for NDVI was shown to be much smaller than that of the moisture indices.

The highest accuracy for the entire growing season was achieved on 22 November with $D_{1650 \mathrm{~nm}}$, but this was only marginally higher than the NDII results. Both were more accurate than the ML classification for that date (table 1). Additionally, accuracy distributions were calculated for all indices by iterating thresholds by 0.001 (figure 3). A wide range of thresholds result in accuracies higher than the ML for each of the moisture indices (figure 3 ). This indicates that the calculation of the

Table 1. Accuracies calculated using thresholds determined from the 10-field training set (denoted Calculated) for 22 November 2000 data are compared to the maximum accuracy (denoted Maximum) achieved for each index by iteratively changing threshold values, and the accuracy attained by classifying six reflective bands using an ML algorithm $(95.48 \%)$. 'Calculated' values in this table are identical to those displayed in figure 2 for this date.

\begin{tabular}{lcccc}
\hline & \multicolumn{3}{c}{ Accuracy $(\%)$} \\
\cline { 2 - 5 } & Calculated & Maximum & Maximum-Calculated & Calculated-ML \\
\hline$D_{1650 n m}$ & 97.74 & 98.07 & 0.33 & 2.26 \\
NDII & 97.42 & 98.07 & 0.65 & 1.94 \\
MSI & 95.48 & 98.07 & 2.59 & 0.00 \\
NDVI & 66.77 & 78.07 & 11.30 & -28.71 \\
\hline
\end{tabular}

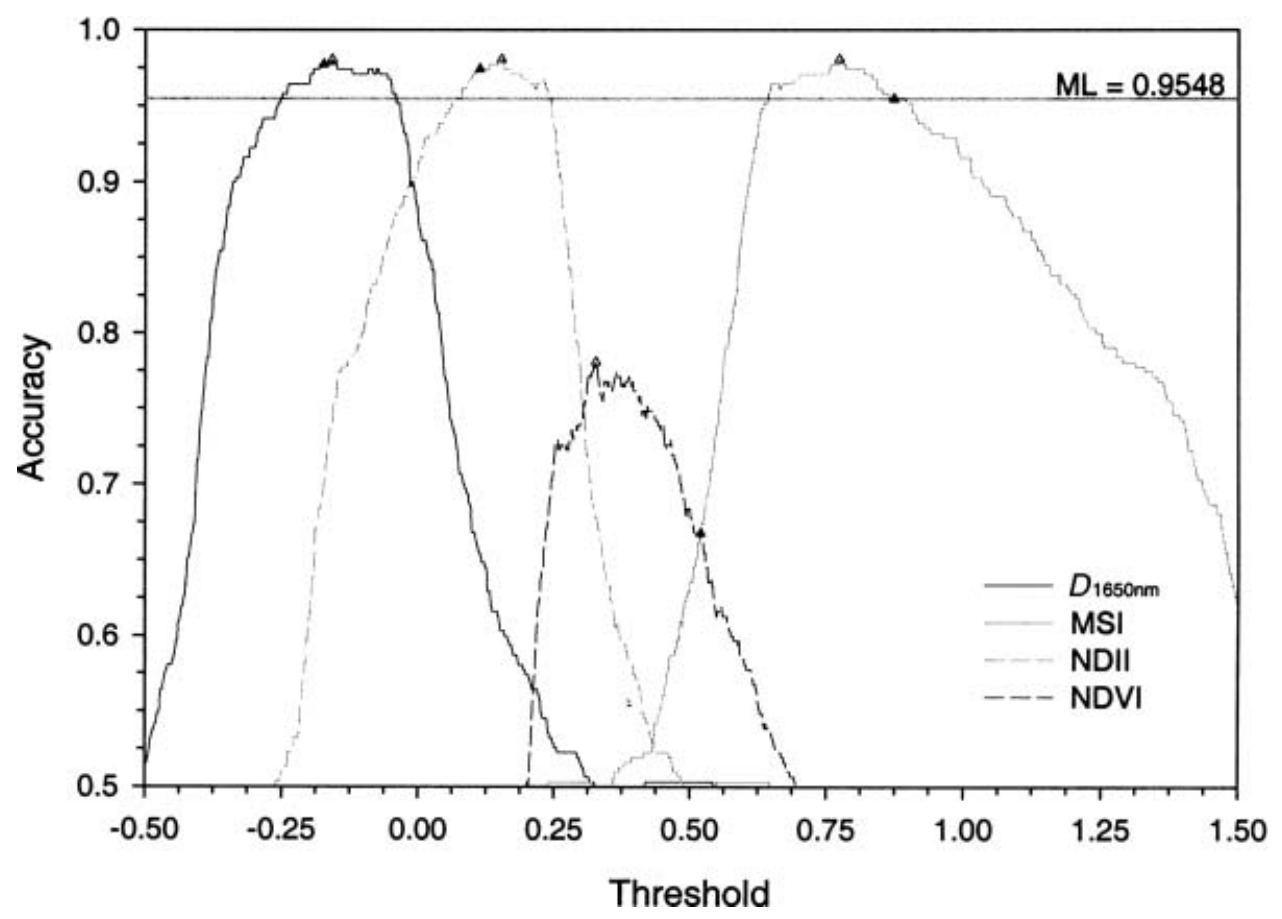

Figure 3. Accuracy distributions (as proportions) for index-based classifications of 22 November data. The grey line is the accuracy achieved by the ML classification $(95.48 \%)$. Filled and open triangles represent Calculated and Maximum accuracies, respectively presented in table 1 . 
threshold is not particularly sensitive, and partly explains why accuracies determined from a small training set were close to the maximum (table 1).

\section{Conclusions}

In general, all three moisture indices were used to classify the data more accurately than the ML algorithm early in the growing season, supporting the hypothesis that targeted spectral feature selection can improve accuracy. ML classification was more accurate than analyses based on the indices after this point, probably due to crop canopy closure and the resultant reduction of between-class environmental moisture variability. As between-class variability was no longer almost entirely due to moisture, the inclusion of ETM + bands 1-3 no longer degraded class discrimination. This difference was greatest late in the season when between-class leaf colour differences were maximized and environmental moisture differences were minimized. Specifically, the two major conclusions from this research are:

1. Rice/non-rice classification accuracy can be improved at specific times by targeting a spectral feature related to environmental moisture.

2. Isolating relevant times when this occurs is vital because the loss of accuracy at other times can be great when compared to standard ML classification.

Since the index-based technique uses only algebraic-type functions, the classification procedure is simplified, resulting in reduced software requirements and increased potential for use in operational applications.

\section{Acknowledgments}

This research was funded by the Cooperative Research Centre for Sustainable Rice Production and CSIRO Land and Water. Many thanks to David Jupp, Lynn Thorp, Monisha Kaul, Leo Lymburner, Catherine Ticehurst, Richard Silberstein, Iain Hume, staff at CICL, Don McCaffery, and four anonymous reviewers, who all improved the quality of this work.

\section{References}

Aplin, P., Atkinson, P. M., and Curran, P. J., 1999, Fine spatial resolution simulated satellite sensor imagery for land cover mapping in the United Kingdom. Remote Sensing of Environment, 68, 206-216.

Barbosa, P. M., Casterad, M. A., and Herrero, J., 1996, Performance of several Landsat 5 Thematic Mapper (TM) image classification methods for crop extent estimates in an irrigation district. International Journal of Remote Sensing, 17, 3665-3674.

Congalton, R. G., 1991, A review of assessing the accuracy of classifications of remotely sensed data. Remote Sensing of Environment, 37, 35-46.

Gausman, H. W., Escobar, D. E., Everitt, J. H., Richardson, A. J., and Rodriguez, R. R., 1978, Distinguishing succulent plants from crop and woody plants. Photogrammetric Engineering and Remote Sensing, 44, 487-491.

Hardisky, M. A., Klemas, V., and Smart, R. M., 1983, The influence of soil salinity, growth form, and leaf moisture on spectral radiance of Spartina alterniflora canopies. Photogrammetric Engineering and Remote Sensing, 49, 77-83.

Hunt, E. R., and Rock, B. N., 1989, Detection of changes in leaf water content using nearand middle-infrared reflectances. Remote Sensing of Environment, 30, 43-54.

KoKAly, R. F., and Clark, R. N., 1999, Spectroscopic determination of leaf biochemistry using band-depth analysis of absorption features and stepwise multiple linear regression. Remote Sensing of Environment, 67, 267-287.

TuCKER, C. J., 1980, Remote sensing of leaf water content in the near infrared. Remote Sensing of Environment, 10, 23-32. 